\title{
Descripción de paso superior vehicular de la Autovía del Cantábrico realizado con materiales compuestos
}

\section{Description of a traffic bridge of the Cantabrian SpeedWay made of composite materials}

\author{
L. M. Mieres ${ }^{(*)}$, I. Calvo(*), A. Miravete(**), E. Gutiérrez(***), E. Shahidi(****), C. López ${ }^{(* * * * *)}$, \\ J. Cuartero(**), P. Comino(******) y R. Guzmán de Villoria(**)
}

Recepción/Received: 14-IX-04

Aceptación/Accepted: 1-XII-05

\section{RESUMEN}

En julio del 2004 se ha finalizado un paso superior vehicular de 46 metros de luz en la Autovía del Cantábrico en el tramo Tamón (Carreño) - Otur (Luarca) en el sub-tramo Vegarrozadas-Soto del Barco, muy próximo al Aeropuerto de Asturias. La peculiaridad de esta obra es que su estructura resistente está formada íntegramente por materiales compuestos. Este paso superior ha sido el resultado del proyecto Iberoeka "Pumacom", iniciado en el año 2003. Un aspecto fundamental en el desarrollo de esta obra ha sido la resolución de las vigas del puente, fabricadas en fibra de carbono/epoxi sobre un bloque de poliuretano. Otro de los desafíos de mayor interés desde el punto de vista de los materiales consistía en la unión entre las vigas en carbono/epoxi y poliuretano con el tablero realizado en hormigón con un armado muy ligero formado por barras de diámetro $16 \mathrm{~mm}$ separadas cada $20 \mathrm{~cm}$. El material seleccionado para los conectores de ambos elementos estructurales fue un material compuesto de fibra de vidrio AR fabricado mediante pultrusión.

Palabras clave: refuerzo, fibra, puente, hormigón, corrosión.

\section{SUMMARY}

In july 2004 a 46 metres length bridge was placed at the Cantábrico Higway between Tamón (Carreño) - Otur (Luarca) that place is located near Asturias airport. This particular bridge has been manufactured mainly with composite materials. This bridge is the final result of an Iberoeka "Pumacom" funded project launched at the beginning of 2003. An innovative issue was the beams of the structure totally manufactured with epoxy/carbon fibre laminated on polyurethane preforms. Another very interesting challenge considered in this project is the joint between longitudinal bridge beams and the supporting platform made of light mortar and steel rods. Joints and connectors were made of AR galls fiber pultruded profiles.

Keywords: reinforcement, fibre, bridge, concrete, corrosion.

\footnotetext{
(*) Departamento de I+D, Necso Entrecanales y Cubiertas S.A., Madrid.

(**) Instituto de Ciencia de Materiales de Aragón, Zaragoza.

(***) JRC-ISIS, Milán, Italia.

(****) Advanced Composites Group, ACG, Reino Unido.

(*****) ICCE Eduardo Torroja, Madrid.

$(* * * * * *)$ Saint Gobain España, Madrid. 


\section{INTRODUCCIÓN}

De los 578.000 pasos superiores de autovías y autopistas de Estados Unidos, 112.000 están considerados como deteriorados, siendo imposible el paso de tráfico al incumplir las normas de seguridad (1). Un ratio similar $-27 \%$ - podría aplicarse al resto del mundo. La causa más importante de este problema es la corrosión de las armaduras metálicas. Cuando se produce un agrietamiento en el hormigón, el agua entra en contacto con las armaduras metálicas ocasionando la corrosión de éstas. Cuando la corrosión se extiende, aumenta el agrietamiento en el hormigón adyacente, generando un ciclo destructivo que provoca un fallo generalizado de la obra.

Como resultado de este problema, hace ya varios años, se ejecutaron numerosas obras introduciendo materiales compuestos de matriz orgánica, los cuales no están sometidos a procesos de corrosión. En particular, durante las dos últimas décadas se han llevado a cabo numerosos puentes en los que se mantenía la estructura resistente tradicional (en hormigón armado o acero) e implantándose el tablero en materiales compuestos. Sin embargo, se ha apreciado en los últimos años un decrecimiento de la aplicación de los materiales compuestos en este campo. De la revisión de los 58 tableros de puentes puestos en obra en Estados Unidos desde 1996, hay varios estudios (2-3) que concluyen que el excesivo coste de los tableros en materiales compuestos puede suponer un inconveniente para el desarrollo de aplicaciones de éstos en la construcción.

La única obra de envergadura documentada que plantea las vigas longitudinales en materiales compuestos es el King Stormwater Channel Bridge en San Diego, que por otra parte incluía también el tablero en fibra de vidrio [4]. Esta obra presenta vigas de sección circular en fibra de carbono rellenas de hormigón. Si bien, ha supuesto un hito en la aplicación de los materiales compuestos en la construcción, su coste ha sido muy elevado y el diseño de la sección de las vigas -importado directamente de la aplicación del refuerzo de columnas de hormigón mediante carbono- es, sin duda, mejorable.

En la línea de realizar un avance en este sector, se inició en nuestro país en el año 2003, el proyecto Iberoeka "Pumacom" liderado por NECSO y con participación de ISPRA (I talia), ACG (Reino Unido) y los socios españoles: IETCC Torroja (Madrid), Sistemas y Procesos Avanzados S.L. (Zaragoza), Saint Gobain (Alcalá de Henares), ICSA (Toledo) y la Universidad de Zaragoza. Este proyecto ya ha conseguido su objetivo más importante al finalizarse el pasado mes de julio el paso superior en la Autovía del Cantábrico (5).

Este paso superior está dividido en cuatro tramos de 10, 13,13 y 10 metros respectivamente, ya que presenta cuatro luces y cinco apoyos (Figura 1). La estructura está formada por tres vigas continuas en sección cajón sobre las que descansa la losa de hormigón.

En la Figura 2 aparece una fotografía de la citada obra. Este paso superior duplica la luz del "Kings Stormwater Channel Bridge", situado en la carretera SR-86 cerca del lago "Salton Sea" en las proximidades de la ciudad californiana de San Diego. Este puente norteamericano presenta una luz de 20,1 metros y tiene un único apoyo en el centro del vano. Las seis vigas longitudinales que constituyen la estructura resistente están basadas en el concepto "Composite Shell System" (4).

A lo largo del proyecto, se han contemplado los siguientes ensayos:

- Ensayos de caracterización de los materiales

- Ensayo de conectores

- Ensayos de uniones

- Ensayos de abolladura

- Ensayo de envejecimiento

- Ensayos de la viga a escala 1:3

- Ensayos de la viga a escala 1:1

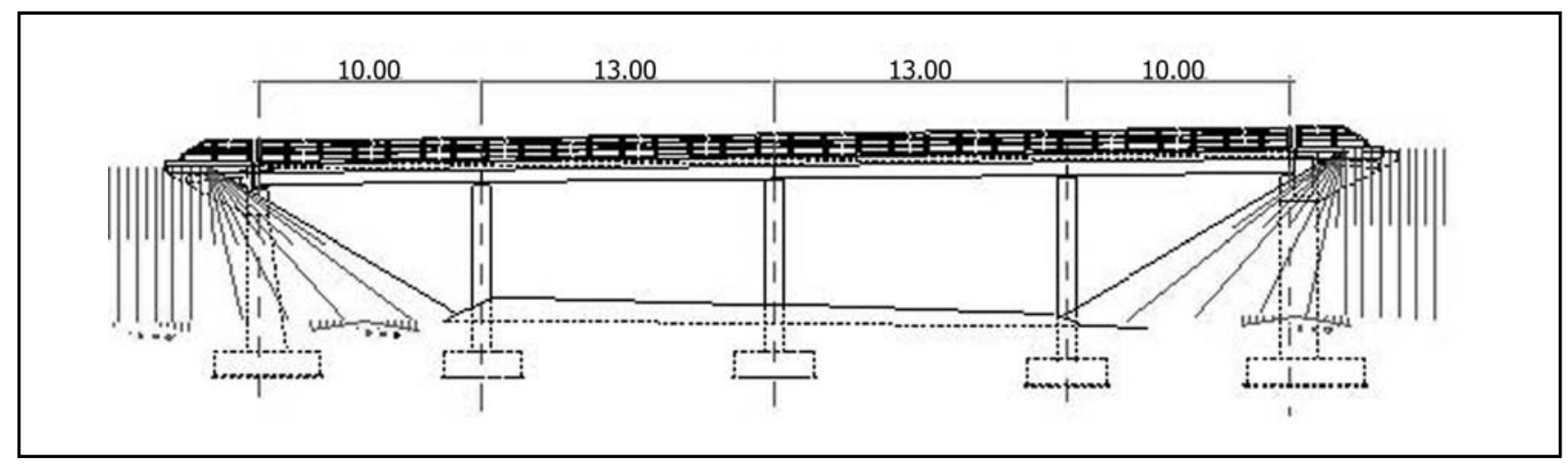

Figura 1. Sección transversal del paso superior. 


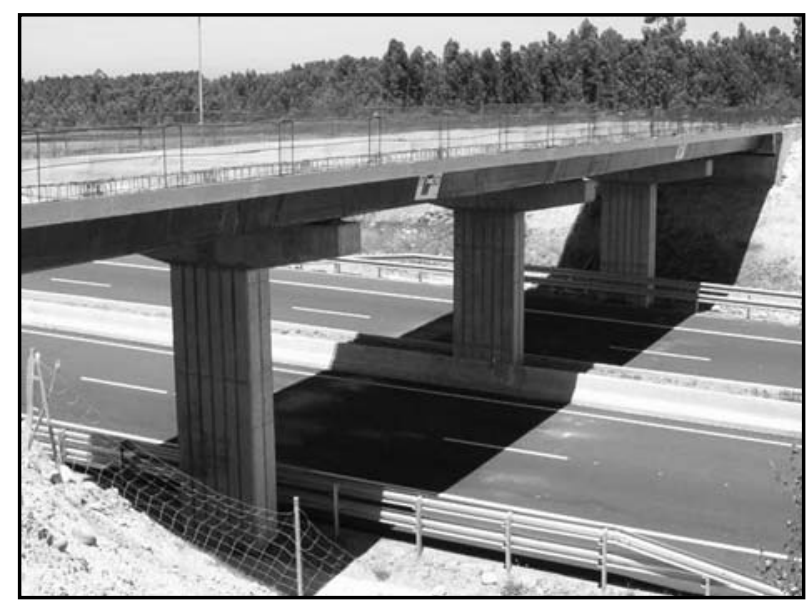

Figura 2. Vista general del paso superior de la Autovía del Cantábrico.

\section{CARACTERÍSTICAS DE LOS MATERIALES EMPLEADOS}

Se ha empleado como materiales estructurales: carbono/epoxi, vidrio AR/poliéster, acero y hormigón. Sus características son las siguientes:

\section{Carbonolepoxi}

- Resistencia longitudinal en dirección $1\left(\mathrm{f}_{1}\right)=1.863,00 \mathrm{MPa}$

- Resistencia longitudinal en dirección $2\left(\mathrm{f}_{2}\right)=33,00 \mathrm{MPa}$

- Módulo de elasticidad longitudinal en dirección 1 $\left(\mathrm{E}_{1}\right)=122,00 \mathrm{GPa}$

- Módulo de elasticidad longitudinal en dirección 2 $\left(\mathrm{E}_{2}\right)=9,1 \mathrm{GPa}$

- Coeficiente de Poisson $\left(v_{12}\right)=0,32$

- Coeficiente de Poisson $\left(v_{21}\right)=0,02$

- Módulo de elasticidad transversal en dirección 12 $\left(\mathrm{G}_{12}\right)=4 \mathrm{GPa}$

- Módulo de elasticidad transversal en dirección 13 $\left(\mathrm{G}_{13}\right)=4 \mathrm{GPa}$

- Resistencia transversal en dirección $12\left(\mathrm{f}_{12}\right)=60,00 \mathrm{MPa}$

- Coeficiente de dilatación térmica en dirección $1 \mathrm{a}_{11}=$

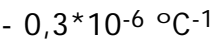

- Coeficiente de dilatación térmica en dirección 2 y $3 a_{2}=$ $a_{3}=28 \times 10^{-6}{\stackrel{0}{ } C^{-1}}^{-1}$

\section{Vidrio AR/poliester}

- Resistencia longitudinal en dirección $1\left(f_{1}\right)=1.050,00$ $\mathrm{MPa}$

- Resistencia longitudinal en dirección $2\left(\mathrm{f}_{2}\right)=31,00 \mathrm{MPa}$

- Módulo de elasticidad longitudinal en dirección 1 $\left(\mathrm{E}_{1}\right)=34,00 \mathrm{GPa}$

- Módulo de elasticidad longitudinal en dirección 2 $\left(\mathrm{E}_{2}\right)=2,9 \mathrm{GPa}$

- Coeficiente de Poisson $\left(v_{12}\right)=0,32$

- Coeficiente de Poisson $\left(v_{21}\right)=0,02$
- Módulo de elasticidad transversal en dirección 12 $\left(\mathrm{G}_{12}\right)=3,8 \mathrm{GPa}$

- Módulo de elasticidad transversal en dirección 13 $\left(\mathrm{G}_{13}\right)=3,8 \mathrm{GPa}$

- Resistencia transversal en dirección $12\left(\mathrm{f}_{12}\right)=38,00 \mathrm{MPa}$

- Coeficiente de dilatación térmica en dirección $1 \mathrm{a}_{11}=$ $15 \times 10^{-6}{ }^{\circ} \mathrm{C}^{-1}$

- Coeficiente de dilatación térmica en dirección 2 y $3 \mathrm{a}_{2}=$ $a_{3}=28 \times 10^{-6}{ }^{\circ} C^{-1}$

\section{Acero estructural}

Las armaduras que se emplean son de tipo Fe 510. Se emplean para estos aceros las siguientes propiedades (material de Saint Venant-Kirchof: material hiperelástico isótropo y homogéneo):

- Módulo de elasticidad, E $=210,00 \mathrm{GPa}$

- Coeficiente de Poisson $v=0,30$

- Masa volumétrica es $7.850 \mathrm{~kg} / \mathrm{m}^{3}$

- El coeficiente isotrópico de dilatación térmica es $a_{11}=$ $12 \times 10^{-6} \stackrel{\circ}{0} \mathrm{C}^{-1}$

- El valor del límite elástico, $\mathrm{f}_{\mathrm{y}}=355 \mathrm{~N} / \mathrm{mm}^{2}$

- El coeficiente parcial de seguridad, 1,15

\section{Hormigón estructural}

Las propiedades consideradas (materiales isótropos y homogéneos) para el hormigón estructural del tablero, de las pilas y de los estribos son (EHE, 1998):

- Módulo de Elasticidad, $\mathrm{E}=28,00 \mathrm{GPa}$

- Coeficiente de Poisson, $v=0,20$

- La masa volumétrica es 2500 kg/m³ (hormigón armado)

- El coeficiente isotrópico de dilatación térmica es $\mathrm{f}_{11}=$ $10 \times 10^{-6}$ o $^{-1}$

- La resistencia característica de proyecto es $\mathrm{f}_{\mathrm{ck}}=25$ $\mathrm{MPa}$

- El coeficiente parcial de seguridad, 1,5

- La resistencia característica a tracción: $\mathrm{f}_{\mathrm{ct}, \mathrm{k}}=0,21$. $\left(f_{c k 2}\right)^{1 / 3}$

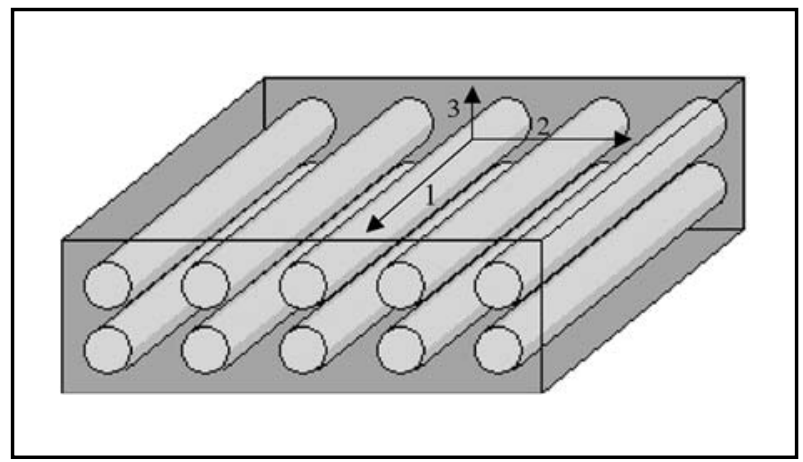

Figura 3. Direcciones en una lámina unidireccional. 


\section{ANÁLISIS DE LA ESTRUCTURA}

La simulación del puente se ha llevado a cabo mediante una malla que consta aproximadamente de 125.000 nodos y 130.000 elementos finitos. Los elementos utilizados han sido de tipo "lámina" para las pieles de fibra de carbono y de tipo "sólido" tanto para simular la espuma de poliuretano, como para el hormigón y los apoyos elásticos de los pilares.

En la Figura 4 se representa la deformada del puente con un factor de amplificación de 20 .

Se han introducido once estados de carga correspondientes a las posibles combinaciones de cargas de viento, nieve y trenes de carga. Los coeficientes de seguridad superaron los límites admisibles en todos los casos de carga y en todos los elementos que componen la obra.

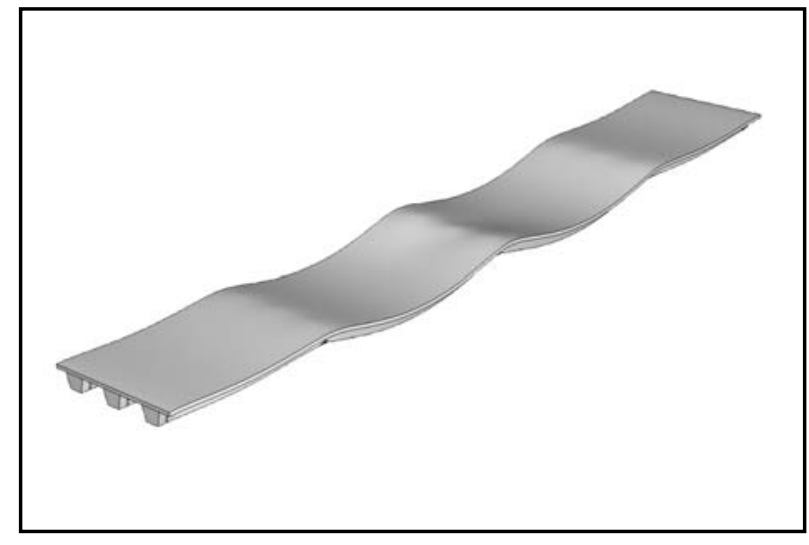

Figura 4. Deformada ampliada del puente.

\section{RESOLUCIÓN DE LAS VIGAS}

El paso superior de la Autovía del Cantábrico no sólo supone un avance en lo que se refiere a su luz, sino a la tecnología empleada. Mientras que el norteamericano King Stormwater Channel Bridge utilizaba secciones circulares, el español incorpora secciones trapeciales, mucho más eficientes, al presentar superiores ratios rigidez/peso y resistencia/peso. El interior de las vigas se resuelve implantando espumas de poliuretano, una solución más eficiente y ligera que el hormigón (Figura 5).

La viga se ensayó, primero a escala 1:3 (6-7). La principal limitación del diseño estructural del puente es la flecha, ya que los requisitos a estados límites últimos los cumple sobradamente, como era de esperar teniendo en cuenta las características mecánicas de estos materiales, que son muy superiores a las de los tradicionales. De ahí que sea especialmente relevante conocer con detalle las flechas que se producen bajo las diferentes combinaciones de cargas.

Con este fin se colocaron rosetas y captadores longitudinales. Con el objetivo de comprobar las solicitaciones reales, se simularon diferentes estados de carga para obtener los esfuerzos determinantes en apoyos y centro de vano. Tras varias medidas de flecha para determinar la rigidez de la viga, ésta se llevó a rotura para evaluar sus modos de fallo y su adecuación con los cálculos realizados hasta el momento. En la Figura 5 aparece una fotografía de la parte inferior del puente donde se pueden apreciar las vigas de fibra de carbono en color negro.

Adicionalmente al objetivo principal, el ensayo que se describe permite cubrir los siguientes objetivos secundarios, pero no por ello carentes de importancia:

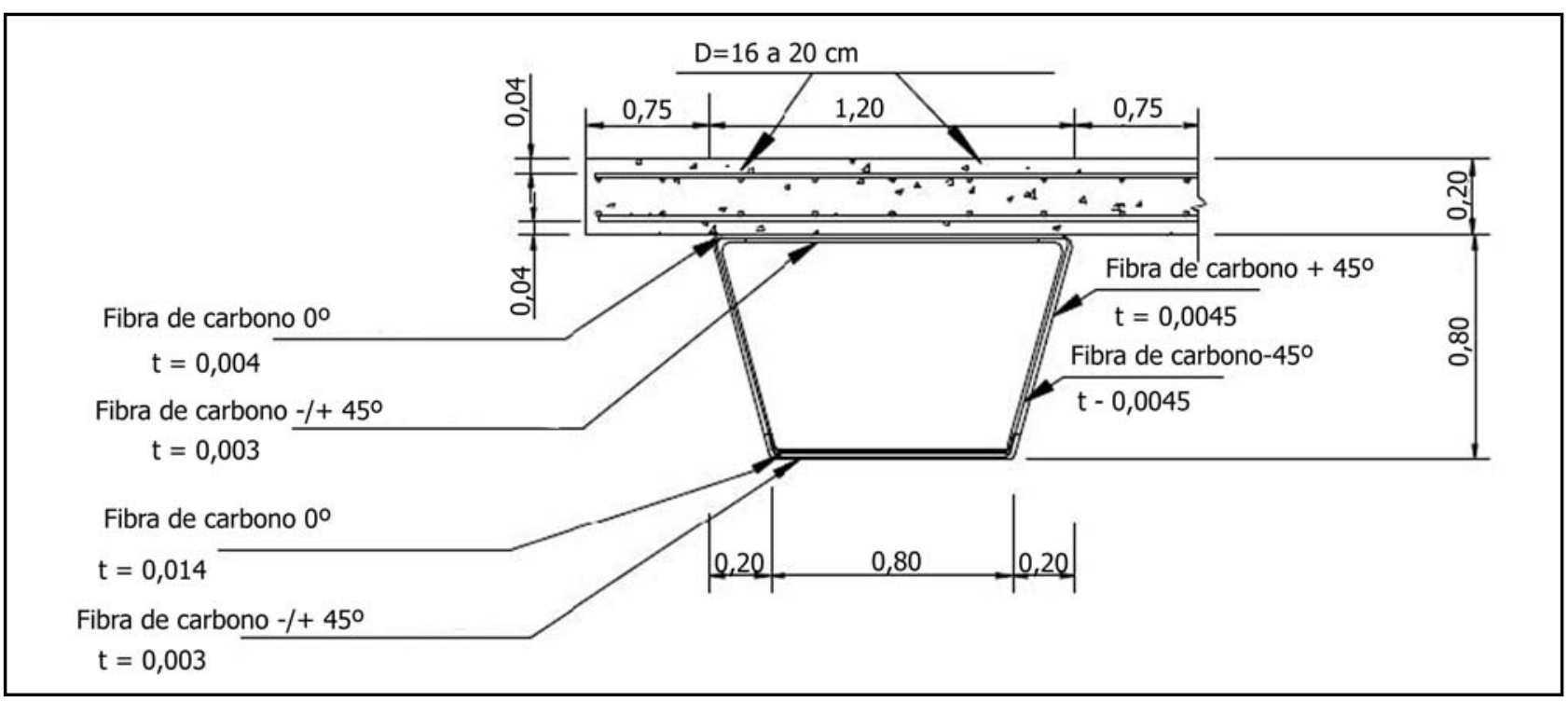

Figura 5. Solución constructiva en el paso superior de Asturias. 


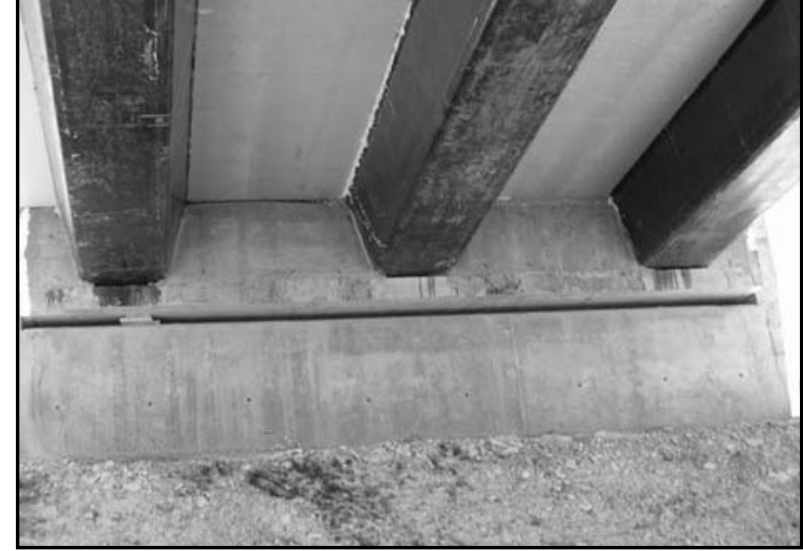

Figura 6. Detalle de las vigas longitudinales del paso superior de la Autovía del Cantábrico en carbono/epoxi y espuma de poliuretano.

- Comportamiento de los conectores entre la losa de hormigón y el cajón de fibra. Los conectores seleccionados son perfiles en doble $T$ de pultrusión que se sitúan en la parte superior de la viga. Los resultados del ensayo nos han permitido analizar el comportamiento solidario de la losa-cajón, así como su capacidad para absorber el esfuerzo rasante.

- Evaluar si los rigidizadores transversales y longitudinales cumplen con su función. Se utilizaron rigidizadores tipo "sándwich" introducidos en el núcleo de poliuretano que sirve de molde para la fabricación de los paneles.

- Comprobar experimentalmente la posibilidad de abolladura en la sección cajón, tanto en el ala inferior debida a compresión simple, como en el alma debida a la concomitancia de cortante y flexión. Previamente se hizo un cálculo analítico que nos sirve de valor de control de los resultados obtenidos.

- Evaluar la aparición de efectos locales en los apoyos o en la interfase hormigón-fibra.

- Estimar un valor de rotura de la viga real para compararlo con el obtenido numéricamente.

En la Figura 7, aparece una fotografía del ensayo a escala 1:3. En resumen, el objeto del ensayo es comprobar experimentalmente los datos obtenidos analíticamente a fin de corregir las posibles deficiencias de la viga real, especialmente a nivel de detalle: rigidizadores, conectores, etc.

Es importante conocer el comportamiento de estos elementos, comunes en el diseño de puentes mixtos de acero-hormigón y que queremos adaptar para los materiales compuestos. Deben dimensionarse correctamente a fin de evitar deficiencias en el comportamiento estructural del conjunto.

Posteriormente se ensayó una viga a escala 1:1 (Figura 8). Las conclusiones de este último ensayo fueron las siguientes:

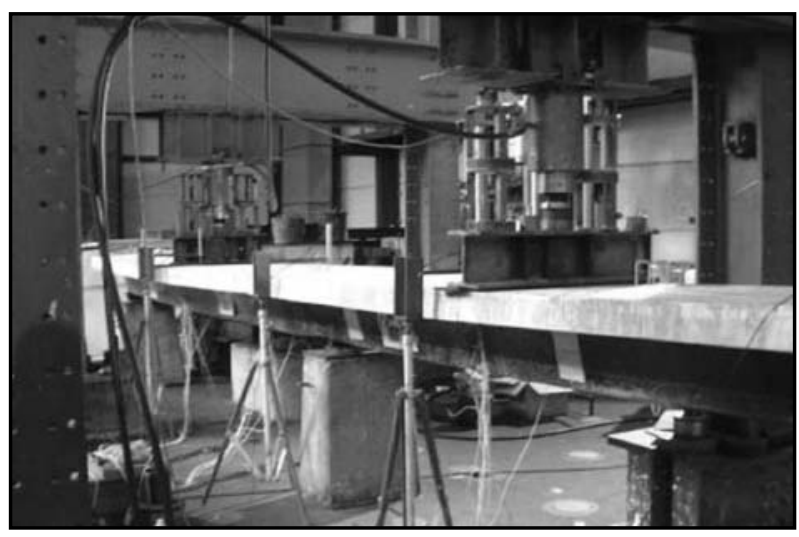

Figura 7. Fotografía del ensayo a escala 1:3.

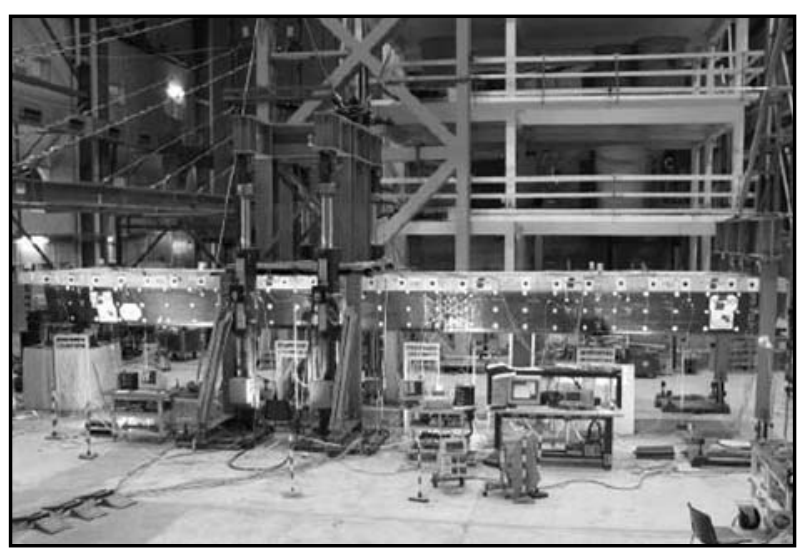

Figura 8. Ensayo y monitorización de las vigas a escala 1:1.

- La viga cumple los requerimientos de rigidez (I/600).

- La unión entre vigas cumple los esfuerzos solicitados en el diseño, porque debido a la tipología del ensayo la sección de unión estuvo solicitada al máximo momento positivo con un cortante concomitante que era superior al máximo cortante negativo. Sabiendo que los esfuerzos en las uniones son muy inferiores a estos máximos que sólo se producen en centros de vano 0 apoyos, podemos asegurar que el comportamiento de las uniones es excelente.

Las solicitaciones resistidas por la estructura fue de 1,6 veces superior a las cargas de diseño mayoradas lo que proporciona un coeficiente de seguridad global de 2,4. Teniendo en cuenta que después de este ensayo se añadieron unos refuerzos en las zonas más solicitadas, se puede suponer que el nuevo coeficiente de seguridad de las vigas que se han colocado en obra es de 3,6 .

Otro aspecto relevante de esta obra consiste en la utilización de conectores fabricados con fibra de vidrio AR (7-9), que incorpora ligereza, prestaciones mecánicas y es resistente frente a los álcalis del hormigón (Figura 9). Dichos conectores están unidos al carbono mediante adhesivo estructural. 


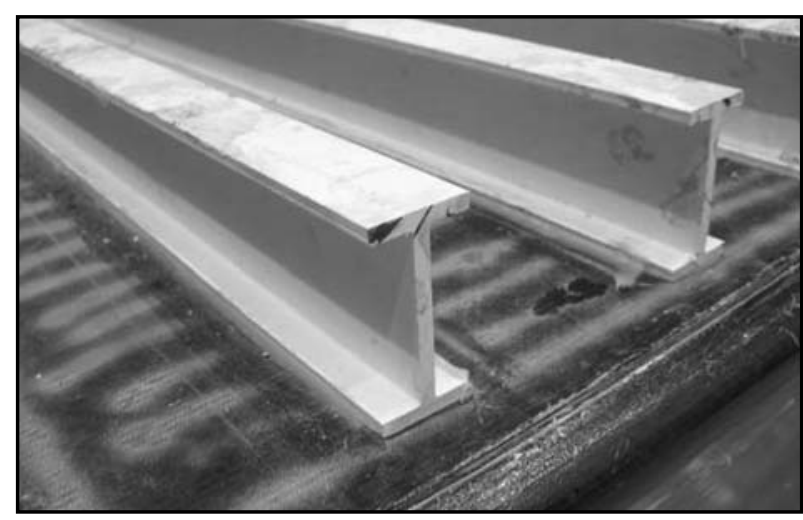

Figura 9. Conectores de fibra de vidrio AR/poliéster adheridos a la viga de carbono/epoxi.

\section{CONCLUSIONES}

Recientemente, se han evaluado los resultados de los puentes realizados hasta la fecha en materiales compuestos y se ha llegado a la conclusión de que el tablero en fibra de vidrio no resulta la solución óptima al grave problema de la corrosión que existe actualmente por un problema de coste.

Otras alternativas como tableros de hormigón soportados por vigas en fibra de carbono están cobrando fuerza en la comunidad científica internacional. En esta línea, se inició en nuestro país en el año 2003 el proyecto Iberoeka "Pumacom", que ha conseguido su objetivo más importante al finalizarse el pasado mes de julio el paso superior en la Autovía del Cantábrico. Este paso superior tiene 46 metros de luz, presenta tres vigas longitudinales en carbono y el tablero en hormigón con un armado muy ligero formado por barras de diámetro $16 \mathrm{~mm}$ separadas cada $20 \mathrm{~cm}$.

La estructura resistente del paso superior del punto kilométrico 1.640 del subtramo Vegarrozadas de la Autovía del Cantábrico, finalizado en julio del 2004 está resuelta con materiales compuestos. Las vigas están fabricadas con carbono/epoxi sobre un núcleo de poliuretano y los conectores en fibra de vidrio AR/poliéster.

A lo largo del proyecto, se han contemplado los siguientes ensayos:

- Ensayos de caracterización de los materiales

- Ensayo de conectores

- Ensayos de uniones

- Ensayos de abolladura

- Ensayo de envejecimiento

- Ensayos de la viga a escala 1:3

- Ensayos de la viga a escala 1:1

Los resultados en todos los casos han sido satisfactorios y se ha finalizado una obra que demuestra la viabilidad de los materiales compuestos para obras de carácter estructural. Este tipo de construcción implica un considerable ahorro en peso de la estructura, una drástica reducción del tiempo de puesta en obra y el aumento de la vida de la obra, al tratarse de materiales no sometidos a procesos de corrosión. Especial énfasis se ha puesto en el aspecto económico, al seleccionar materiales y procesos que permiten competir con el hormigón armado con las consiguientes ventajas de disminución de costes de transporte, puesta en obra y mantenimiento.

\section{BIBLIOGRAFÍA / BIBLIOGRAPHY}

(1) Black, S.: "Are hybrid designs the future for composite bridge decks?", febrero (2004), p. 20.

(2) Black, S.: "How Are Composite Bridges Performing?", Composites Technology, diciembre (2003), p. 16.

(3) Turner, M. K.; Harries, K. A.; Petrou, M. F. y Rizos, D.: "In situ Structural Evaluation of a GFRP Bridge Deck System", Composite Structures, vol. 65 (2004), pp. 157-165.

(4) Burgeño, R.; Davol, A. y Seible, F.: “The Carbon Shell System for Modular Bridge Components", Second International Conference on Composites in Infrastructure, Tucson, Arizona, enero (1998).

(5) Mieres, J. M.; Calvo, I.; Gutiérrez, E.; Shahidi, E.; Miravete, A, López, C.: "The Spanish Pumacon 46 m long bridge: A cost competitive alternative", Proceedings of $4^{\text {th }}$ International Conference on Advanced Composite Materials in Bridges and Structures, Calgary, Canada, 2004.

(6) Gutiérrez, E.; Di Salvo, G.; Mieres, J. M.: "Experimental results from laboratory tests on an 8 metre beam manufactured from hybrid composite formwork", Advanced Composite letters, vol. 7, 6, pp. 155-160.

(7) Mieres, J. M.; Gutiérrez, E.; Bautista, S.; Calvo I.: "Hybrid beam of $8 \mathrm{~m}$ length manufactured with concrete and composites materials", Hormigón y Acero, 230 (2003).

(8) Miravete, A.; Mieres, J. M.; Calvo, I. et al.: "Experimental study on AR fiberglass connectors for bridges made of composite materials", Mater. Construcc., 56 (282): 77-82, abril-junio de 2006.

(9) Miravete, A.; Mieres, J. M.; Calvo, I. et al.: "Behaviour of AR glass fibre for building structural applications", Mater. Construcc., 55 (280): 63-69, octubre-diciembre de 2005. 